\title{
Drosophila-Antenne gewährt Einblicke in grundlegende Mechanismen des Hörens
}

\author{
Martin C. Göpfert
}

\begin{abstract}
Zusammenfassung
Hörorgane sind faszinierende Sinnesorgane: Sie detektieren Schall und können gelegentlich Schall produzieren. Diese spontanen otoakustischen Emissionen sind eines der Hauptindizien für die Existenz des so genannten cochleären Verstärkers, eines aktiven Verstärkungsmechanismus im Innenohr, der auf Haarzellmotilität beruht. Insekten haben weder Cochlea noch Haarzellen. Neuere Untersuchungen an den antennalen Hörorganen von Drosophila zeigen allerdings, dass es auch in der FruchtfliegenAntenne einen ,cochleären" Verstärker gibt: Wie die Haarzellen von Wirbeltieren erzeugen die auditorischen Sinnesneuronen in der Drosophila-Antenne aktiv Kräfte und unterstützen dadurch die winzigen, durch Schall induzierten Schwingungen, die sie transduzieren. Diese neuronale Krafterzeugung, ihr Einfluss auf das makroskopische Verhalten des Ohrs und die beteiligten molekularen Mechanismen sind Thema dieses Artikels, der einen Überblick über den derzeitigen Kenntnisstand zur Funktionsweise des Fruchtfliegen-Ohrs gibt. Parallelen zwischen Fliegen-Hören und Wirbeltier-Hören werden aufgezeigt, welche die Drosophila-Antenne für die Analyse grundlegender Hörmechanismen prädestinieren.
\end{abstract}

\begin{abstract}
Summary
Drosophila antenna provides insights into fundamental mechanisms in hearing. Ears are fascinating organs: they detect sound and occasionally produce sound. These spontaneous otoacoustic emissions provide the most compelling evidence for the existence of the cochlear amplifier, an active amplificatory process inside the cochlea that resides in the motility of inner-ear hair cells. Insects neither have a cochlea nor hair cells, yet recent studies on the antennal ear of Drosophila show that a 'cochlear' amplifier also exists in the antennae of flies: like hair cells, the auditory sensory neurons in the Drosophila antenna actively generate forces to augment the minute vibrations they transduce. This neuron-based force-generation, its impact on the ear's macroscopic performance, and the molecular mechanism that bring about this force-generation are the topics of this article, which summarizes our knowledge about the functional workings of the Drosophila ear. Parallels between hearing in flies and vertebrates are described that recommend Drosophila for studying fundamental mechanisms in hearing.
\end{abstract}

Key words: chordotonal organ; cochlear amplifier; hearing; insect antenna; mechanosensory transduction.

\section{Einleitung}

Die vollständige Genom-Sequenz der Fruchtfliege Drosophila melanogaster wurde im Jahr 2000 publiziert (Adams et al. 2000). Das Genom wurde anschließend nach Genen durchforstet, deren menschliche Homologa in Krankheiten impliziert sind. 548 Gene wurden gefunden, die bei 714 menschlichen Krankheiten eine Rolle spielen (Reiter et al. 2001). In einem begleitenden Kommentar bemerkte Lawrence T. Reiter, der Erstautor dieser Studie: ,This came as a bit of a surprise as most people don't think to study hearing or cancer in Drosophila' (University of California Press Release, 15.06.2001). In Hinblick auf das Hören scheint diese Bemerkung gerechtfertigt: Die Fruchtfliege hat zwar ein Ohr, dieses Ohr hat jedoch mit dem menschlichen Ohr zumindest äußerlich nicht viel gemein (Abbildung1). Neuere Untersuchungen bringen jedoch überraschende molekulare und funktionelle Parallelen zwischen Fliegenhören und menschlichem Hören ans Licht, die das Fliegenohr zur Untersuchung grundlegender Mechanismen des Hörens qualifizieren. Diese Parallelen sind Thema dieses
Artikels, der einen Einblick in den Stand der Fruchtfliegen-Hörforschung gibt.

\section{Hören mit der Antenne: Das Drosophila-Ohr}

Wie wir selbst kommuniziert auch Drosophila mittels akustischer Signale. Die Details dieser Kommunikation sind zugegebenermaßen etwas eigen: Nähert sich ein Fliegenmännchen einem Weibchen, zuckt es einen seiner Flügel in einem regelmäBigen Muster und produziert dadurch ein ,Liebeslied' (Greenspan und Ferveur 2000). Dieses Lied erhöht die Kopulationsbereitschaft des Weibchens und animiert umstehende Männchen zum Singen, sodass eine regelrechte Fliegenparty resultiert.

Die Wahrnehmung der Fliegengesänge vermittelt die Fliegenantenne (Abbildung 1 A, B). Das keulenförmige dritte Antennensegment und seine seitliche Arista bilden den Schallempfänger (Göpfert und Robert 2001). Sie entsprechen damit funktionell unserem Trommelfell. Bei Beschallung dreht sich dieser antennale Schallempfänger um das zweite Antennensegment, wie ein Schlüssel in seinem Schloss (Göpfert und Robert 2001). Das zweite Antennensegment enthält ein auditorisches Sinnesorgan, das Johnstonsche Organ. Dieses chordotonale Streckrezeptororgan umfasst ca. 500 primäre mechanosensorische Neuronen (Kamikouchi et al. 2006). Die Entwicklung dieser Neuronen wird durch dasselbe Gen initiiert, das die sensorischen Haarzellen in unserem Ohr spezifiziert (Hassan und Bellen 2000). Die Neuronen sind bipolar mit einem proximalen Axon und einem distalen, ciliären Dendriten (Abbildung 1 C). Die Dendriten sind über extrazelluläre Kappen unmittelbar mit dem Schallempfänger verbunden. Schallinduzierte Schwingungen werden folglich direkt auf die mechanosensorischen Neuronen übertragen und von diesen mit Hilfe von Mechanotransduktionskanälen in elektrische Antworten übersetzt. Die Empfindlichkeit der Neuronen ist erstaunlich. Kombinierte mechanische und elektrophysiologische Messungen zeigen, dass das Fliegenohr elektrisch antwortet, sobald Schall die Antennenspitze um ca. 20 Nanometer hin und her bewegt (Albert et al. 2007a; Effertz und Göpfert, in Vorb.). Diese Auslenkung entspricht einer Winkeldrehung von einem viertausendstel Grad. Das Riesenrad am Prater in Wien würde sich bei gleicher Winkeldrehung um etwa zwei Millimeter bewegen, eine Kinderschaukel um lediglich etwa einen zehntel Millimeter. Die sensorischen Neuronen im Fliegenohr und die sensorischen Haarzellen in unserem 
Ohr entwickeln sich unter Kontrolle identischer Gene und wetteifern hinsichtlich ihrer Empfindlichkeit.

\section{Aktive Schwingungsverstärkung im Fliegenohr}

Die Schwingungen einer Kinderschaukel lassen sich durch Anstoßen der Schaukel aktiv verstärken. Mithilfe desselben Tricks erreicht unser Ohr seine Empfindlichkeit - durch Schall induzierte Schwingungen werden im Inneren des Ohrs aktiv unterstützt. Diese Schwingungsverstärkung beruht auf Haarzellmotilität: Wirbeltierhaarzellen reagieren auf Auslenkung ihres sensorischen Haarbündels sowohl elektrisch als auch mechanisch, entweder in dem sie Ihren Zellkörper kontrahieren oder mit ihrem Haarbündel zucken. Durch diese selbst-generierten Bewegungen unterstützen Haarzellen aktiv diejenigen durch Schall ausgelösten Schwingungen, die sie in elektrische Signale transduzieren (Fettiplace und Hackney 2006). Diese mechanische Rückkopplungsverstärkung - gemeinhin bekannt als der cochleäre Verstärker (Ashmore und Gale 2004) - erklärt zwei der erstaunlichsten Eigenschaften unseres Ohrs: 1. Nichtlinearität: Kleine, durch leisen Schall ausgelöste Schwingungen werden maximal verstärkt. Die Folge ist, dass sich die mechanische Empfindlichkeit unseres Ohres nichtlinear mit der Schallintensität ändert - je leiser der Schall, desto empfindlicher wird unser Ohr. 2. Spontane Schallemissionen: Positive Rückkopplungsverstärkung ist störungsanfällig; wird die Verstärkung zu groß, schwingt das System auf. Rückkopplungsschwingungen aufgrund exzessiver mechanischer Verstärkung erklären die erstaunliche Fähigkeit unseres Ohrs, nicht nur Schall zu detektieren, sondern gelegentlich Schall zu emittieren. Solche ,spontanen otoakustischen Emissionen" belegen eindrücklich die Existenz eines aktiven Schwingungsverstärkers in unserem Ohr.

Mechanische Untersuchungen an der Drosophila-Antenne haben überraschenderweise gezeigt, dass es auch im Fliegenohr einen aktiven Schwingungsverstärker gibt (Göpfert und Robert 2003; Göpfert et al. 2005, 2006) (Abbildung 2). Wie die Mechanik unseres Ohrs verbessert sich die Mechanik des antennalen Schallempfängers der Fliege nichtlinear mit der Schallintensität: Je leiser der Schall ist, desto größer wird die mechanische Empfindlichkeit des antennalen Schallempfängers, ein Effekt, der sich mit Verschlechterung des physiologischen Zustands der Fliege verliert

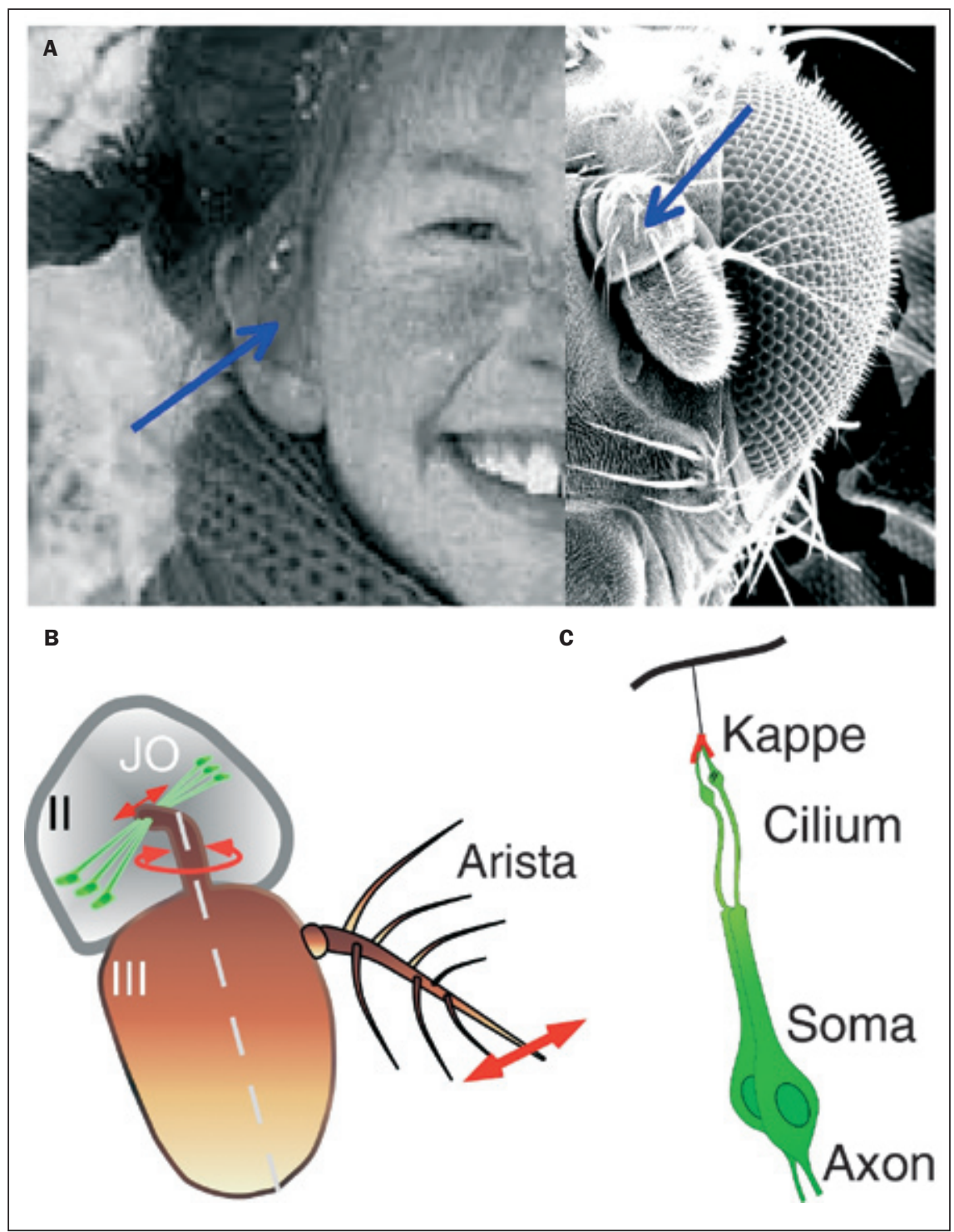

Abb. 1: Fliegenohr. (A): Menschliches Ohr (links, blauer Pfeil) und Fruchtfliegenohr (rechts, blauer Pfeil) haben äußerlich wenig gemein. (B): Bei der Fliege dienen das dritte, distale Antennensegment (III) und die federartige Arista als Schallempfänger. Die auditorischen Neuronen des Johnstonschen Organs (JO) im zweiten Antennensegment (II) setzen von zwei Seiten direkt an der Basis des Schallempfängers an (modifiziert nach Albert et al. 2007b). Bei Beschallung rotiert der Schallempfänger um seine Längsachse vor und zurück, wodurch die Neuronen des Johnstonschen Organs alternierend gedehnt und gestreckt werden. (C): Die auditorischen Neuronen sind primäre Sinneszellen mit einem distalen sensorischen Cilium und einem proximalen Axon. Eine extrazelluläre Kappe verbindet jeweils zwei bis drei Neuronen mit dem Schallempfänger (modifiziert nach Göpfert 2007).

(Göpfert und Robert 2003; Göpfert et al. 2006). Gelegentlich schwingt das System auch auf. Dann schwingt die Antenne von selbst - in Abwesenheit externer akustischer Reize (Göpfert und Robert 2003; Göpfert et al. 2005; Stoop et al. 2006). Die zwei wesentlichen Kennzeichen des cochleären Verstärkers - Nichtlinearität und selbsterhaltende Rückkopplungsschwingungen - zeigen sich im Ohr einer Fliege.

\section{Schwingungsverstärkung durch neuronale Motilität}

Die Klärung des zellulären Ursprungs mechanischer Aktivität im Fliegenohr hat die Fruchtfliegen-Genetik erleichtert. Im Rahmen genetischer Screens wurden zahlreiche genetische Defekte identifiziert, die Taubheit bei Fliegen verursachen (Kernan et al. 1994; Eberl et al. 1997). Eines der 
betroffenen Gene codiert das Protein NompA (= No mechanoreceptor potential A). NompA ist ein Tektorin verwandtes extrazelluläres Matrixprotein, das bei der Fliege den antennalen Schallempfänger mit den Sinneszellen im Inneren des Ohrs verbindet. Wird NompA durch NullMutationen im zugrunde liegenden Gen eliminiert, trennt sich der Schallempfänger von den auditorischen Sinneszellen ab - es resultiert ein „konduktiver"Hörverlust, die Übertragung im Ohr wird unterbrochen (Chung et al. 2001). Diese genetische Sek- tion führt zum Verlust der Nichtlinearität der Antennenmechanik und zum Verlust spontaner Antennenoszillationen (Göpfert und Robert 2001; Göpfert et al. 2005). Bei Isolation von den auditorischen Neuronen verhält sich der antennale Schallempfänger von Drosophila folglich linear und passiv; Nichtlinearität und mechanische Aktivität sind Eigenschaften der Sinneszellen im Ohr. Dieser Verdacht wird durch den Verlust von Nichtlinearität und spontanen Oszillationen bei tilB (= touch insensitive larva $B)$ - und btv (= beethoven) - Mutanten unterstützt

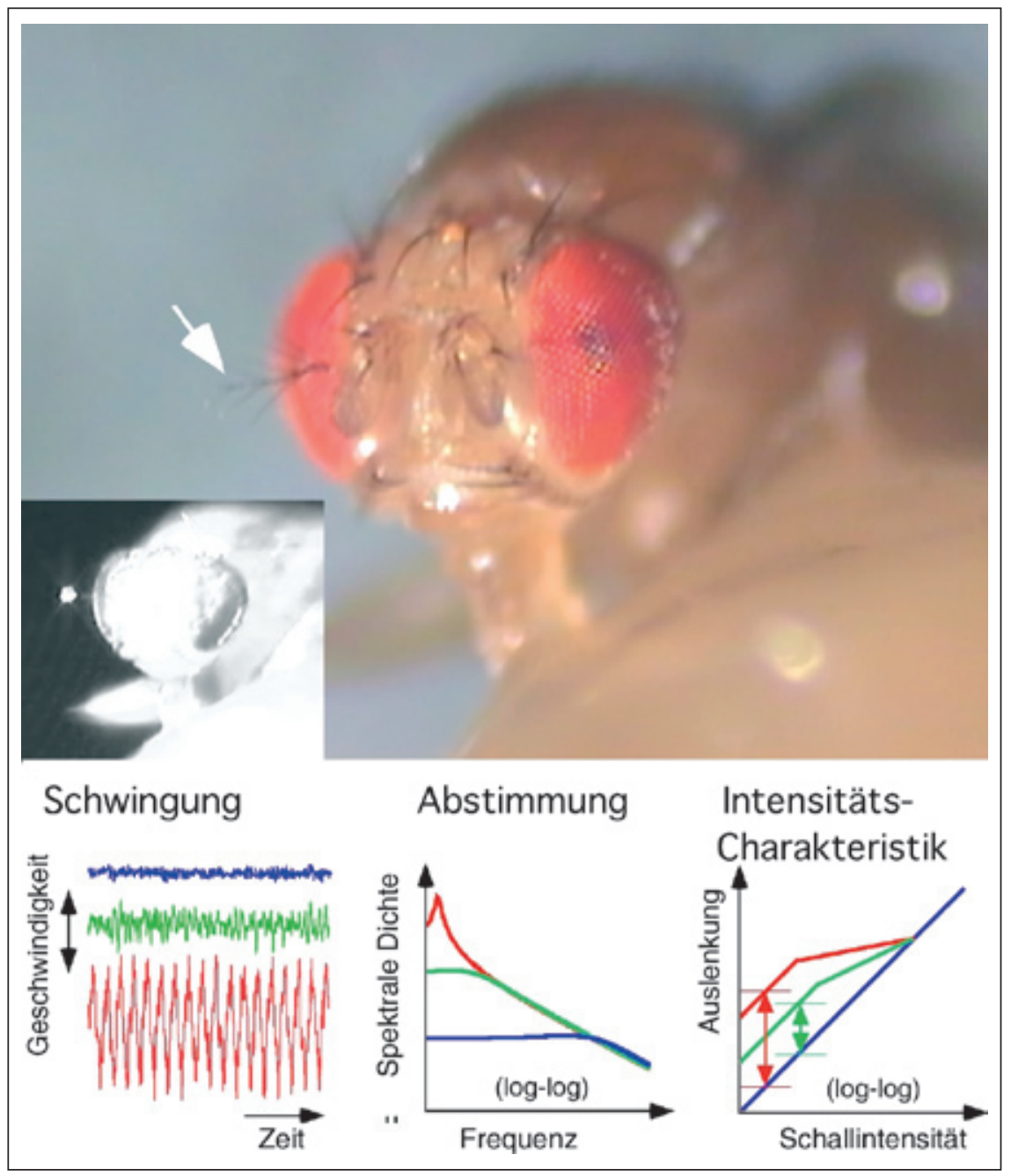

Abb. 2: Mechanik des Fliegenohrs. Oben: Die Bewegungen des antennalen Schallempfängers lassen sich an der Spitze der Arista berührungsfrei mittels eines Laser-Doppler-Vibrometers messen. Der auf die Aristaspitze fokussierte Laserstrahl ist in der Detailansicht zu sehen. Unten: Zeitspuren der Aristaschwingungen in Abwesenheit externer Reize (links), entsprechende Frequenzspektren (Mitte) und Intensitätscharakteristik (rechts). Beim toten Tier (blau) zeigt die Arista nur thermisches Rauschen (links) und ist breit abgestimmt (Mitte). Bei Beschallung an der Bestfrequenz steigt die Schwingungsamplitude linear mit der Schallintensität (rechts). Mechanische Rückkopplung erhöht im lebenden Tier (grün) die Antennenfluktuationen, verbessert die Frequenzabstimmung und moduliert die Intensitätscharakteristik in nichtlinearer Weise. Gelegentlich wird die Rückkopplung zu groß, es kommt zu selbsterhaltenden Oszillationen (rot) (modifiziert nach Albert et al. 2006).
(Göpfert und Robert; Göpfert et al. 2003). Mutationen in $b t v$ und tilB beeinflussen die strukturelle Integrität der dendritischen Cilien der mechanosensorischen Neuronen im Fliegenohr. Mutationen in $b t v$ führen zu strukturellen Aberrationen der ,ciliary dilation', einer Schwellung im distalen Teil des Ciliums (Eberl et al. 2000). Mutationen in $t i l B$ wiederum führen zum Verlust ciliärer Dyneinarme und, infolgedessen, zu Spermienimmotilität (Eberl et al. 2000). Der kombinierte Verlust aktiver Verstärkung bei diesen Mutanten verdeutlicht, dass der Verstärker im Drosophila-Ohr, analog Haarzell getriebenen cochleären Verstärker von Wirbeltieren, auf mechanischer Aktivität mechanosensorischer Neuronen beruht (Abbildung 3).

\section{Neuronaler Energiebeitrag}

Bislang haben wir gesehen, dass Ohren sich nichtlinear verhalten und Schwingungen generieren. Der Nachweis aktiver Verstärkung erfordert jedoch den Nachweis von Energiegewinn - mehr Energie muss aus einem Verstärker rauskommen als ursprünglich hineingesteckt wird. Bei der Fruchtfliege ließ sich ein solcher Energiegewinn erstmals für ein intaktes $\mathrm{Ohr}$ belegen (Göpfert et al. 2005). Es stellte sich heraus, dass die Neuronen von intakten Fliegen die mechanische Energie des Schallempfängers im Mittel um 19 zepto Joule $\left(19 \times 10^{-21}\right.$ Joule) anheben. Dies entspricht etwa einem Zwanzigstel der Energie eines einzelnen grünen Photons. Wird die aufgebrachte Energiemenge im Fliegenohr auf $200 \mathrm{zJ}$ erhöht, schwingt das System auf. Offensichtlich sind die Neuronen im Fliegenohr in der Lage, die bereitgestellte Energiemenge zu justieren: Bei zu kleiner Energie gibt es keine Verstärkung, bei zu großer Energie schwingt das System auf. Zwischen diesen beiden Zuständen prognostiziert die Theorie einen kritischen Punkt, eine Hopf-Bifurkation, an dem die Empfindlichkeit maximal ist (Ospeck et al. 2001). Wie die Haarzellen im Wirbeltierohr maximieren die Sinneszellen im Fliegenohr möglicherweise ihre Empfindlichkeit durch Ausnutzen dieses kritischen Punkts.

\section{Molekulare Basis aktiver Verstärkung}

Welcher molekulare Mechanismus liegt der neuronalen Motilität und der aktiven Verstärkung im Fliegenohr zugrunde? Bei Wirbeltieren scheinen zwei verschiedene Mechanismen eine Rolle zu spielen: (i) eine Prestin vermittelte Elektromotilität und (ii) das Zusammenspiel zwischen Mechano- 
transduktionskanälen und Adaptationsmotoren (Fettiplace und Hackney 2006). (i) Prestin vermittelte Elektromotilität ist anscheinend auf die äußeren Haarzellen der Säugercochlea beschränkt (Dallos und Fakler 2002; Albert et al. 2007c). Diese Zellen kontrahieren und relaxieren ihr Soma in Abhängigkeit des Membranpotenzials, sie sind elektromotil. Molekular beruht diese Form aktiver Haarzellbewegung auf dem Protein Prestin, einem modifizierten Aniontransporter, der Spannungsänderungen in Zellbewegungen übersetzt, vermutlich, indem das Protein seine Konformation in spannungsabhängiger Weise ändert (Dallos und Fakler 2002). Das Zusammenspiel zwischen Transduktionskanälen und Adaptationsmotoren wiederum scheint der aktiven Motilität der sensorischen Haarbündel von Haarzellen zugrunde zu liegen, eine Form aktiver Zellbewegung, die sowohl Säuger- als auch bei Nichtsäugerhaarzellen zeigen. Beide Mechanismen könnten auch im Fliegenohr eine Rolle spielen: Ein Prestin Homolog wird im auditorischen Organ der Fliege exprimiert (Weber et al. 2003). Laufende Untersuchungen deuten allerdings darauf hin, dass dieses Homolog keine Rolle bei der aktiven Schwingungsverstärkung im Fliegenohr spielt (Fritz, Albert, Eberl und Göpfert, unveröffentl.). Hinsichtlich des zweiten Mechanismus - das Zusammenspiel zwischen Transduktionskanälen und Adaptationsmotoren - sieht die Sache besser aus. Wie im Folgenden dargestellt, führt uns die Untersuchung dieses Mechanismus zu Ionenkanälen im Fliegenohr und letztendlich zur auditorischen Transduktion.

\section{Aktive Verstärkung und TRP-Kanäle}

Die Wandlung mechanischer Schwingungen in elektrische Signale im Inneren des Ohrs erfolgt durch spezifische Mechanotransduktionskanäle. Zumindest in Wirbeltierhaarzellen werden diese Ionenkanäle direkt durch mechanische Reize aktiviert (Le Masurier und Gillespie 2005). Auditorische Transduktionskanäle sind sehr rar und konnten bislang weder bei Insekten noch bei Wirbeltieren molekular identifiziert werden. Bei der Fliege wurden allerdings Kanalproteine beschrieben, die möglicherweise die Transduktion im Ohr vermitteln. Es handelt sich um Kationkanäle der „Transient Receptor Potential“ (TRP)-Familie: NompC (= TRPN1) und die TRPVs Nan (= Nanchung) und Iav (= Inactive). NompC wurde zuerst in taktilen Borsten der Fliege entdeckt (Walker et al. 2000). Null-Mutationen im nompC-Gen

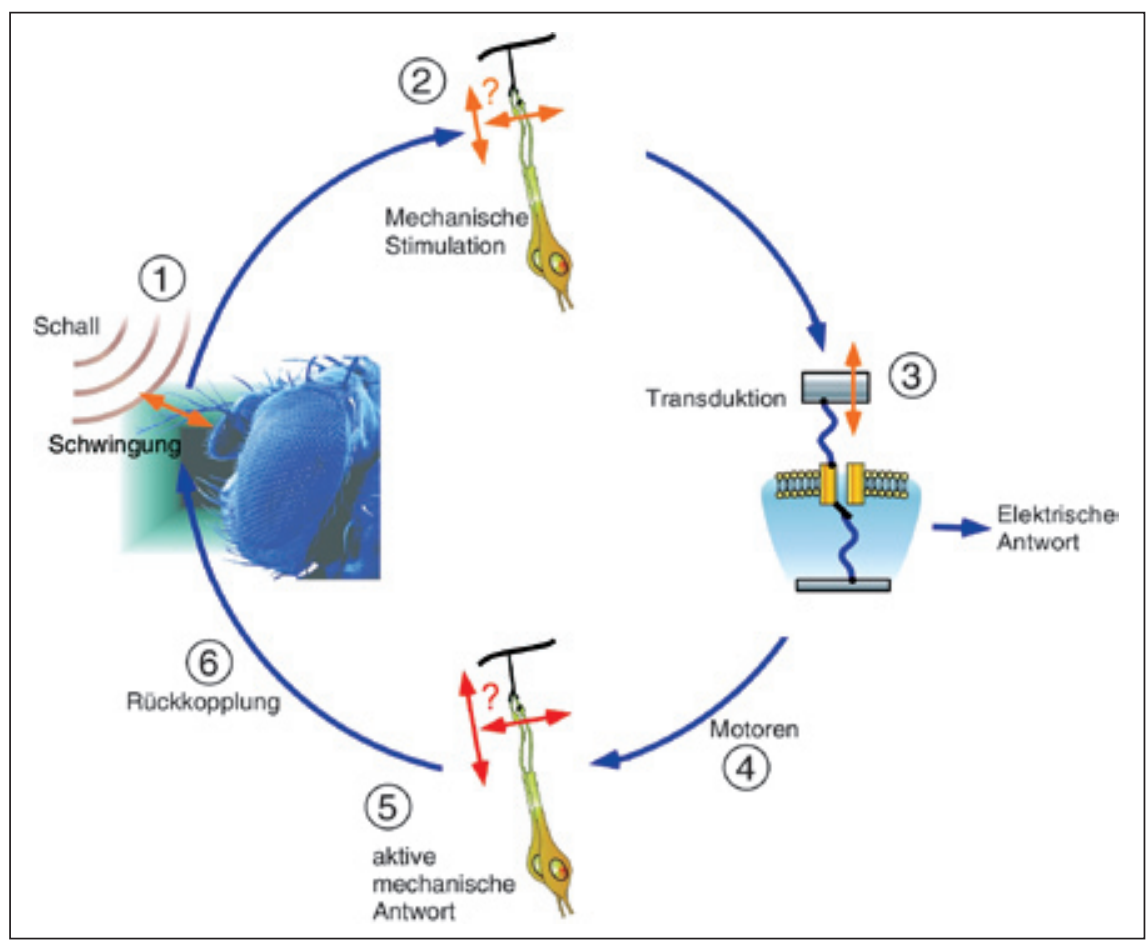

Abb. 3: Mechanische Rückkopplungsverstärkung im Fliegenohr. Schallinduzierte Schwingungen des antennalen Schallempfängers (1) werden auf die Neuronen des Johnstonschen Organs übertragen und führen zu Längsänderungen oder lateralen Bewegungen der Dendriten (2). Diese Bewegungen modulieren die Öffnung von Transduktionskanälen (3), welche die Bewegungen in elektrische Antworten wandeln. Zusätzlich kommt es zur Aktivierung von molekularen Motoren (4), die die Zelle mechanisch treiben (5). Diese aktiven Zellbewegungen werden in die Antennenschwingungen positiv rückgekoppelt (6) - die Antennenschwingungen werden aktiv verstärkt (modifiziert nach Göpfert 2007).

eliminieren dort mechanisch evozierte, adaptierende Ströme und ein schwächeres nompC-Allel beschleunigt die Adaptation. Auch im Fliegenohr gibt es NompC. Dort reduzieren sich akustisch evozierte Nervantworten um ca. die Hälfte, wenn NompC fehlt (Eberl et al. 2000). Ein vollständiger Verlust dieser Nervantworten tritt auf, werden Nan oder Iav genetisch eliminiert (Kim et al. 2003; Gong et al. 2004). Diese TRPVs bilden einen voraussichtlich heteromultimeren Nan-Iav-Kanal im Cilium der auditorischen Neuronen. Analysen der Antennenmechanik zeigen, dass NompC, Nan und Iav wesentliche Rollen bei der aktiven Verstärkung im Fliegenohr spielen (Göpfert et al. 2006): Wird der NompC-Kanal eliminiert, geht die aktive Schwingungsverstärkung verloren. Der gegenteilige Effekt - eine Förderung der Verstärkung - tritt auf, wenn Iav oder Nan fehlen: In diesem Fall erhöht sich der durch aktive Verstärkung bewirkte Empfindlichkeitsgewinn von ca. 10 (Kontrollen) auf 100 (nan- und iav-Mutanten), d.h., die Verstärkung wird exzessiv. Die Folge sind Rückkopplungsschwingungen
- die Antenne von nan- und iav-Mutanten oszilliert kontinuierlich von selbst. Während NompC für die Verstärkung benötigt wird, mindern Nan und Iav folglich die Verstärkung und verhindern dadurch Rückkopplungsschwingungen im Ohr. Diese Verstärkungskontrolle erfolgt über NompC: Sind sowohl Nan als auch NompC defekt, treten keine Rückkopplungsschwingungen auf und die aktive Verstärkung geht verloren (Göpfert et al. 2006). Sind NompC und Nan-Iav Transduktionskanäle? Zumindest Nan-Iav scheint dies nicht zu sein: Rückkopplungsverstärkung impliziert Kenntnis des Eingangssignals; sind die Transduktionskanäle defekt, sollte folglich die aktive Verstärkung verloren gehen. NompC, nicht aber Nan-Iav, könnte demnach ein auditorischer Transduktionskanal sein. Die reduzierten Nervantworten bei NompC-Null-Mutanten müssten dann von einem zusätzlichen, NompC-unabhängigen Transduktionskanal stammen. Nan-Iav wiederum scheint neben der Verstärkungskontrolle auch der Fortleitung elektrischer Signale vom Ort der Transduktion zum auditorischen Nerven zu dienen. Dies erklärt 
voraussichtlich, warum die Nervantwort bei nan- und iav-Mutanten vollständig fehlt (Abbildung 4).

\section{Von der Verstärkung zum Transduktionsmechanismus - Aktivierung der Transduktionskanäle im Fliegenohr}

Analysen der Antennenmechanik belegen die TRP-Abhängigkeit aktiver Verstärkung im Fliegenohr. Die Identität der Transduktionskanäle und deren Beitrag zur Verstärkung im Fliegenohr bleiben jedoch unklar, solange sich der Transduktionsmechanismus nicht direkt untersuchen lässt. Glücklicherweise spiegelt sich auch dieser Mechanismus in der Mechanik der Fliegenantenne wieder und lässt sich somit nichtinvasiv analysieren: Bewegungen der Fliegenantenne aktivieren die Transduktionskanäle direkt, wobei sich dieses Öffnen und die Adaptation der Kanäle in der Antennenmechanik widerspiegeln (Albert et al. 2007a, b). Analysen dieser mechanischen Signaturen zeigen, dass die Transduktionskanäle in den auditorischen Neuronen der Fliege - wie die Transduktionskanäle in Wirbeltierhaarzellen - über Federn aktiviert werden und vermutlich mit Adaptationsmotoren assoziieren (Albert et al. 2007a, b). Aufgrund der experimentellen Zugänglichkeit der DrosophilaAntenne lassen sich jetzt zwei Fliegen mit einer Klappe schlagen: Einerseits kann jetzt gezielt nach Komponenten der Transduktionsmaschinerie gefahndet werden, und anderseits lässt sich der Beitrag der Transduktion zur aktiven Verstärkung im intakten Ohr quantifizieren. Dies ist Gegenstand laufender Untersuchungen. Was sich dabei abzeichnet, ist, dass das Zusammenspiel zwischen Transduktionskanälen und Adaptationsmotoren, welches der Haarbündelmotilität von Wirbeltierhaarzellen zugrunde liegt, aktive Verstärkung im Fliegenohr erklärt.

\section{Konvergenz oder Konservierung auditorischer Mechanismen?}

Hören bei Fliege und Mensch beruht auf verblüffend ähnlichen Mechanismen. Sind diese Mechanismen konserviert oder sind sie konvergent entstanden? Insekten und Wirbeltiere haben unabhängig Ohren entwickelt, die mechanosensorischen Zellen in ihren Ohren stammen jedoch aller Wahrscheinlichkeit nach von einer gemeinsamen Vorläuferzelle ab (Fritzsch und Beisel 2004). Dies erklärt das Vorkommen identischer genetischer Programme für Entwicklung

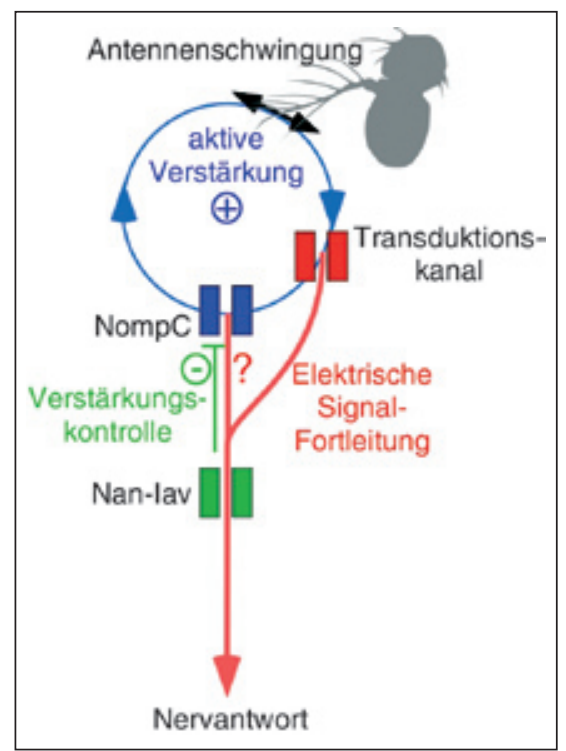

Abb. 4: Modell der TRP-Abhängigkeit aktiver Verstärkung im Fliegenohr. NompC (=TRPN1) ist essenzieller Bestandteil der mechanischen Rückkopplungsschleife, die durch Schall ausgelöste Antennenschwingungen aktiv verstärkt. Der TRPV - Kanal Nan-lav wirkt unterhalb von NompC und reduziert das Ausmaß der NompC-abhängigen Rückkopplungsverstärkung. NompC ist möglicherweise ein Transduktionskanal, es müssen jedoch weitere Transduktionskanäle (rot) existieren, da der Verlust von NompC die Nervantwort nur teilweise eliminiert. Nan-lav wird anscheinend für die Fortleitung elektrischer Signale unterhalb der Transduktion benötigt, da bei Verlust dieses Kanals die Nervantwort vollständig verloren geht (modifiziert nach Göpfert et al. 2006).

und mechanosensorische Zellfunktion in Wirbeltierhaarzellen und auditorischen Insekten-Neuronen. Inwieweit auch die Maschinerien für Transduktion und Verstärkung konserviert sind, muss sich noch zeigen: NompC, den derzeit besten auditorischen Transduktionskanalkandidat der Fliege, beispielsweise, gibt es in den Haarzellen von Zebrafisch und Frosch, während der Kanal bei Säugern anscheinend vollständig fehlt (z.B. LeMasurier und Gillespie 2005). Nan und Iav-verwandte TRPV-Kanäle gibt es in Säugerhaarzellen, welche Rolle diese Kanäle im Säugerohr spielen, ist allerdings noch nicht geklärt. Selbst wenn Transduktion und Verstärkung in Haarzellen und Fliegenhörzellen auf unterschiedlichen Proteinen beruhen sollten, macht die funktionelle Äquivalenz der auditorischen Transduktions- und Verstärkungsmechanismen in Wirbeltier- und
Fliegenohren letztere für die Hörforschung interessant.

\section{Literatur}

Albert, J.T., Nadrowski, B. und Göpfert, M.C. (2007a): Mechanical signatures of transducer gating in the Drosophila ear. Current Biology 17: 1000-1006.

Ashmore, J. und Gale, J. (2004): The cochlear amplifier. Current Biology 14: R403-R404.

Göpfert, M.C., Humphris, A.D.L., Albert, J.T., Robert, D. und Hendrich, O. (2005): Power gain exhibited by motile mechanosensory neurons in Drosophila ears. Proceedings of the National Academy of Sciences USA 102: 325-330.

Göpfert, M.C., Albert, J.T., Nadrowksi, B. und Kamikouchi, A. (2006): Specification of auditory sensitivity by Drosophila TRP channels. Nature Neuroscience 9: 999-1000.

LeMasurier, M. und Gillespie, P.G. (2005): Hair-cell mechanotransduction and cochlear amplification. Neuron 48: 403-415.

Eine ausführliche Literaturliste kann beim Autor angefordert werden.

\section{Danksagung}

Der Autor dankt den Mitarbeitern der VolkswagenStiftungs-Nachwuchsguppe am Zoologischen Institut der Universität zu Köln und allen externen Kooperationspartnern für die Zusammenarbeit und anregende Diskussionen. Die Arbeiten werden von der VolkswagenStiftung und dem BMBF im Rahmen des Bernstein-Partnerprogramms unterstützt.

\section{Kurzbiographie}

Martin Göpfert studierte Biologie and der Universität Erlangen-Nürnberg. Anschließend arbeitete er als Stipendiat des DAAD und der Deutschen Akademie der Naturforscher Leopoldina an der Universität Zürich und als Royal Society University Research Fellow an der Universität Bristol. Seit Ende 2003 leitet er die VolkswagenStiftungs-Nachwuchsgruppe, Active Auditory Mechanics in Insects' am Zoologischen Institut der Universität zu Köln.

\section{Korrespondenzadresse}

PD Dr. Martin Göpfert

VolkswagenStiftungs-Nachwuchsgruppe

Zoologisches Institut

Universität zu Köln

Weyertal 119

$50923 \mathrm{Köln}$

Tel.: +49 (0) 2214703102

Fax: +49 (0) 2214704889

E-Mail:m.gopfert@uni-koeln.de 